\title{
Multiplicación in vitro de piña MD-2 (Ananas comosus (L.) Merril) haciendo uso de fertilizantes comerciales
}

\author{
Juan Francisco Cuéllar Zometa ${ }^{1}$ - Carlos Eduardo Somoza Vargas $^{2}$ \\ Docentes investigadores, Facultad de Arquitectura e Ingeniería \\ Universidad Católica de El Salvador, El Salvador
}

Recepción: 11/07/2015

Aceptación: 10/08/2015

\section{Resumen}

Este estudio consistió en la multiplicación in vitro de piña MD-2 (Ananas comosus (L.) Merril) bajo la técnica de cultivo de tejidos vegetales, empleando el medio de cultivo desarrollado por Murashige \& Skoog (1962), modificado con $3 \mathrm{mg}$ de 6 bencil amino purina por litro de medio, tratamiento uno (TI). El tratamiento anterior ha sido contrastado con otro que se basa en un terreno de cultivo in vitro, a base de fertilizante comercial hidrosoluble, medio de cultivo que se identificó como «UNICAES 2», tratamiento dos (T2); buscando igualar la concentración y disponibilidad de cada uno de los elementos necesarios para el correcto desarrollo de las plantas.

Se seccionaron yemas apicales de retoños de la base de plantas madre, y se desinfectaron con hipoclorito de sodio al 5\% por 15 minutos, adicionando 3 gotas de tween 80 por cada $100 \mathrm{ml}$ de solución desinfectante. Se seccionaron las yemas de aproximadamente $0.5 \mathrm{~cm}$ de longitud por $0.5 \mathrm{~cm}$ de diámetro. Estas se inocularon en frascos de vidrio conteniendo $30 \mathrm{ml}$ de medio de cultivo con 20 repeticiones por tratamiento. Las yemas se incubaron a 16-8 horas luz-oscuridad; con una temperatura promedio de $28^{\circ} \mathrm{C}$ y 232 pie candela de intensidad lumínica, proporcionada por 3 lámparas fluorescentes. Luego de 40 días se compararon las yemas obtenidas en ambos tratamientos, tomando en cuenta su longitud y número de brotes laterales.

Palabras clave: in vitro, Murashige \& Skoog, fertilizante comercial hidrosoluble, piña MD-2, yemas apicales.

\begin{abstract}
This study consisted of an in vitro multiplication of MD -2 pineapple (Ananas comosus (L.) Merril) under the technique of plant tissue culture, using the culture developed by Murashige \& Skoog (1962), modified with 3mg 6 benzyl purine amino per half liter, treatment one (T1). This treatment has been contrasted with another one that is based on an in vitro culture, based on water-soluble commercial fertilizer, culture that was identified as «UNICAES 2» treatment two ( T2 ); trying to match the concentration and availability of each needed element for proper plant development.

Apical buds were cut from the base of the plant, and disinfected with $5 \%$ sodium hypochlorite for 15 minutes, adding 3 drops of Tween 80 per $100 \mathrm{ml}$ of disinfectant solution. The apical buds of approximately $0.5 \mathrm{~cm}$ long by $0.5 \mathrm{~cm}$ diameter were cut. These were inoculated in glass bottles containing $30 \mathrm{ml}$ of culture with 20 replications per treatment. The buds were incubated at $16 / 08$ hours (light-dark); with an average temperature of $28^{\circ} \mathrm{C}$ and 232 foot-candle of light intensity, provided by three fluorescent lamps. After 40 days the buds obtained in both treatments, were compared in length and number of lateral buds.
\end{abstract}

Key words: in vitro, Murashige \& Skoog, water-soluble commercial fertilizer, MD -2 pineapple, apical buds.

1. Ingeniero agrónomo; email: jfcuellar@catolica.edu.sv

2. Ingeniero agrónomo; email: somoza.vargas@gmail.com 


\section{Introducción}

La piña (Ananas comosus (L) Merril) es una planta monocotiledónea, herbácea y perenne, originaria de América del Sur, de la región de Mattogroso, entre Uruguay y Brasil (Dirección General de Investigación y Extensión Agrícola. Ministerio de Agricultura y Ganadería. San José, Costa Rica, 1991). Aunque actualmente se encuentra muy diseminada por las regiones tropicales, siendo ahora un cultivo de alto valor comercial y nutritivo.

Según Ministerio de Agricultura y Ganadería, El Salvador (1991):

La inflorescencia contiene entre cien y doscientas flores colocadas en forma espiral, fusionadas al eje central. La floración se prolonga entre treinta y sesenta días aproximadamente; el fruto maduro se obtiene ciento treinta y cinco días después de haber emergido la flor. (p 1).

Sajquim Pedro Julio Pac 205

La flor, que da nacimiento a un pequeño fruto bien individualizado en principio lo que se conoce como "baya", es del tipo trímero. Con la bráctea subyacente y comprende: tres sépalos, tres pétalos, seis estambres situados en dos verticilos, un pistilo tricarpelar con ovario ínfero. Los pétalos liguliformes, azul -púrpura, tienen una base blancuzca y llevan sobre su cara daxial las escamas carnosas cuya variedad de forma contribuye a la clasificación de las especies del género Ananás. El conjunto de la corola forma un tubo alargado, ligeramente más ancho en su extremidad y en el centro del cual emergen los tres estigmas violeta pálido del estilo. Tres glándulas nectaríferas desembocan por conductos diferentes en las base del estilo. Las flores son auto estériles, como es corriente en la mayoría de los cultivares, por lo que los óvulos no quedan formados pero por polinización cruzada puede producirse fecundación y formación de pepitas redondas, pequeñas y muy duras. (p. 6)

Cerrato (2013) refiere que:

La piña MD2 es un híbrido que fue desarrollado para satisfacer a un mercado en busca de dulzura excepcional, así como la uniformidad y consistencia en tamaño y madurez. Por medio del Pineapple Research Institute (PRI) en el programa de mejoramiento de la piña se desarrollaron algunos híbridos que tenían numerosas características mejor que la Cayena Lisa, pero no dieron ninguna indicación de que un cultivo se había producido que transformaría el mundo fresco de la industria de exportación de frutas ya que todos ellos tenían defectos que les impedían reemplazar Cayena Lisa. A pesar de esta 
revisión, se identificaron dos selecciones, 73-50 y 73-114 resultante del cruce entre dos híbridos PRI, 58-1184 y 59-443, que son mezclas complejas de diferentes variedades, cada una de más de 50\%, Cayena Lisa. En 1981, pasó a llamarse 73-50 MD-1 para uso interno y 73-114 fue nombrado MD-2. Ambos fueron nombrados por Millie, la esposa de Frank Dillard, entonces director general de la empresa de piña. MD-2 ha sustituido la Cayena Lisa en todo el mundo a excepción de algunas pequeñas áreas de producción en África. En la actualidad se cultiva en varios países como Ecuador, Panamá, Guatemala, Honduras y México y Costa Rica. (p. 2)

La piña MD2 goza de poder ser exportada a Estados Unidos libre de arancel. El comercio de la piña es representativo principalmente por las importaciones. El volumen de importación de piñas aumento de en el 2006 y en el 2008 (29\% y 16\% respectivamente). Sin embargo, para 2009 se redujo un 31.5\% (Alfaro, MAG 2011). El Salvador es incapaz de satisfacer la demanda local por este fruto; dicho esto, tampoco ha alcanzado la capacidad de exportar el mismo a mercados internacionales para generar ingresos económicos.

El sistema de propagación que se da a través de retoños o hijuelos, tiene la desventaja de que el material seleccionado baje la calidad en cada ciclo de cultivo, la contaminación de plagas y enfermedades son heredadas de sus plantas madres e incluso este método de propagación induce a elevar los costos de producción (Suárez 2011). Por eso, métodos de propagación y multiplicación de cultivos más eficientes son necesarios para elevar el nivel técnico de trabajo empleado en la piña, los métodos de base tradicional de trabajo se han demostrado ineficaces a la hora del trabajo con el mismo; la suma de estos factores ha generado un desánimo por parte de los productores agrícolas a iniciar la empresa de trabajar con piña.

La piña es una bromeliaceae, la cual se propaga vegetativamente, presentando un porcentaje de multiplicación muy lento. En la actualidad ha adquirido gran importancia comercial a nivel mundial por la demanda de su fruto. Esto ha llevado a la búsqueda de nuevos métodos de propagación, que permitan acelerar la producción de propágulos para la siembra en el campo entre los cuales cabe señalar el cultivo in vitro que permite mejorar sustancialmente el proceso de multiplicación (Casale y García, 1997).

El Salvador tiene presencia exportadora mínima de fruta con empresas como Dole y Del Monte, los contratos de exportación con empresas de dicho nombre exigen el cumplimiento de ciertos requisitos, dentro de los cuales se encuentra el de proveer suficiente fruto de forma constante; y que este fruto sea 
de la calidad esperada por ellos, para comercializarla en el mercado internacional. Estos requisitos son difíciles de alcanzar por los pocos productores de piña nacionales y por la falta de tecnología que garantice producto de alta calidad. Existe un interés mundial por el cultivo de la piña, pero nuestro país no es capaz de suplir esa necesidad ya que no contamos con la tecnificación suficiente para un acelerado empleo de dicho cultivo en las cantidades necesarias.

El aumento de producción de plantas ha sido una problemática constante en el sector agrícola, para darle resolución a la misma se han diseñado diversos métodos para acelerar la multiplicación, que han sido utilizados a los largo de la historia y han ido evolucionando de múltiples formas. Uno de los métodos más efectivos para lograr una acelerada propagación de cultivos, teniendo cuidado en mantener su calidad genética, es el método de cultivos in vitro.

Según L. A. Mroginski y W. M. Roca (1991):

La amplitud de la definición de «cultivo de tejidos» y los numerosos objetivos que éstos persiguen, constituyen serios escollos en cualquier intento de generalización sobre los factores que afectan el establecimiento de tales cultivos in vitro.

El cultivo de tejidos in vitro comprende, en su acepción amplia, un heterogéneo grupo de técnicas mediante las cuales un explante (parte separada de un vegetal, por ejemplo: protoplasto, célula, tejido, órgano) se cultiva asépticamente en un medio de composición química definida, y se incuba en condiciones ambientales controladas.

Los objetivos buscados por el cultivo de tejidos in vitro son varios pero se pueden resumir así: a) estudios básicos de fisiología, genética, bioquímica y ciencias afines; b) bioconversión y producción de compuestos útiles; c) incremento de la variabilidad genética; d) obtención de plantas libres de patógenos; e) propagación de plantas; y f) conservación e intercambio de germoplasma. (p. 20)

Con respecto a lo anterior, nuestra investigación busca la propagación de plantas de piña MD-2, sumándole la implementación de un terreno de cultivo in vitro a base de productos fertilizantes comerciales; contrastando los resultados con los obtenidos con un terreno de cultivo tradicional.

Ahora bien, hasta las técnicas más innovadoras han sufrido cambios que han permitido su evolución, y el método de cultivos in vitro no ha sido la excepción. La creación y evolución de los terrenos de cultivo in vitro ha ido acorde a los requerimientos nutricionales de cada cultivo, esto ha funcionado bien históricamente; pero revisando los Laboratorios 
que se dedican a estas actividades, desde un punto de vista corporativo-empresarial, se hace necesario también agregarle un nuevo factor a la evolución y creación de los nuevos terrenos de cultivo. Este nuevo aspecto es el de «eficiencia económica», en otras palabras, la rentabilidad.

Las innovaciones técnicas que se deben tomar para mejorar la rentabilidad económica de los procesos in vitro, no deben dejar de lado su principal misión: asegurar que los explantes, establecidos y desarrollados en fases anteriores, sean capaces de soportar y sobrellevar las posibles condiciones adversas a las que se enfrentarían en fases in vitro como en campo abierto.

El terreno de cultivo UNICAES 2, desarrollado en el Laboratorio de Tejidos Vegetales de la Universidad Católica de El Salvador cumple a cabalidad el aspecto económico en el ahorro de recursos; y ha sido sometido en esta investigación para determinar su funcionalidad con respecto a su aporte nutricional en piña MD-2 (Ananas comosus L. Merril) en desarrollo de yemas para multiplicación, siendo a la vez contrapuesto con el conocidísimo terreno de cultivo Murashige \& Skoog.

Según L. A. Mroginski y W. M. Roca (1991):

El primer objetivo en la preparación de un medio de cultivo es suministrar los nutrimentos minerales en concentracio- nes adecuadas. Se deben incluir los macroelementos ( $\mathrm{C}, \mathrm{H}, \mathrm{O}, \mathrm{P}, \mathrm{K}, \mathrm{N}, \mathrm{S}, \mathrm{Ca} \mathrm{Y}$ $\mathrm{Mg}$ ) y los microelementos (B, Zn, Mn, $\mathrm{Cu}, \mathrm{Mo}, \mathrm{Fe}, \mathrm{Cl})$. (p. 44).

El terreno de cultivo in vitro UNICAES 2 cumple a cabalidad el aporte de macro y micro elementos necesarios para un correcto desarrollo de las plantas; también se realizaron los cálculos correspondientes de las cantidades de dichos elementos en el medio.

Los elementos de los cuales parte el terreno de cultivo in vitro UNICAES 2, productos agrícolas como fertilizantes comerciales, garantizan una considerable reducción en los costos de producción de terreno de cultivo con respecto a otros como el Murashige \& Skoog. Otro factor a favor es el fácil acceso a estos productos, evitando inconvenientes como la solicitud de autorización requerida para la adquisición de reactivos de laboratorio, utilizados en otros terrenos de cultivo; también no se está sujeto a la disponibilidad de estos productos por parte de unos pocos distribuidores.

\section{Materiales y métodos}

\section{a. Introducción del material}

Se hizo el trabajo de multiplicación de establecimiento de yemas de hijos de piña MD2 , sometiendo los tratamientos a condiciones ambientales controladas. Todas las actividades realizadas y descritas se llevaron a cabo 
en el Laboratorio de Tejidos Vegetales de la Universidad Católica de El Salvador.

\begin{tabular}{|c|c|}
\hline \multicolumn{2}{|c|}{ Materiales } \\
\hline Descripción & Cantidad \\
\hline Material Vegetal & \\
\hline $\begin{array}{l}\text { Yemas de piña } \\
\text { MD-2 extraídas de } \\
\text { hijos de piña. }\end{array}$ & 30 inicialmente \\
\hline Reactivos & \\
\hline $\begin{array}{l}\text { Reactivos } \\
\text { Medio M\&S }\end{array}$ & 1L/Reactivo \\
\hline Fitohormonas & 3mgBAP/1Lmedio \\
\hline Abonos Comerciales & Detallados en receta \\
\hline Vitaminas & $1 \mathrm{~mL} /$ Litro de medio \\
\hline Phytagel & 5.8g/Prueba \\
\hline MioInositol & $0.2 \mathrm{~g} /$ Prueba \\
\hline $\begin{array}{l}\text { Soluciones } \\
\text { de calibración }\end{array}$ & $15 \mathrm{~mL}$ \\
\hline Equipo & \\
\hline Pipeta & 2 \\
\hline Beakers & 3 \\
\hline Herlen Meyer & 2 de $2 \mathrm{~L}$ \\
\hline & $1 \mathrm{de} 1 \mathrm{~L}$ \\
\hline Cilindro Graduado & 1 \\
\hline PH Meter & 1 \\
\hline Hot Plate & 2 \\
\hline Autoclaves & 2 \\
\hline $\begin{array}{l}\text { Cámaras de } \\
\text { flujo Laminar }\end{array}$ & 1 \\
\hline $\begin{array}{l}\text { Frascos de vídrio } \\
\text { de } 8 \mathrm{oz}\end{array}$ & 50/prueba \\
\hline Tapaderas plásticas & 50/prueba \\
\hline Plástico de embalaje & 1 rollo \\
\hline Insumos & - \\
\hline Electricidad & - \\
\hline Gas & - \\
\hline
\end{tabular}

\section{b. Establecimiento}

El establecimiento de los explantes se llevó a cabo de forma similar. Para esta fase se utilizaron los reactivos y metodología correspondiente a la del terreno de cultivo in vitro Murashige \& Skoog, con variación para establecimiento de piña. Las yemas centrales de los hijos de piña se sometieron a desinfección con hipoclorito de sodio al $20 \%$ por 20 minutos, esto con el fin de eliminar agentes infecciosos inoculados en las muestras; y se aplicó Tween 80 para eliminar la película cerosa.

Dentro de la cámara de flujo laminar, se retiró el hipoclorito de sodio con ayuda de agua desmineralizada con pretratamiento de ósmosis inversa. El establecimiento se realizó a razón de un explante por frasco, para reducir el porcentaje de pérdida por contaminación.

\section{c. Desarrollo del terreno de cultivo UNICAES 2}

Como período de desarrollo de los explantes, después de la fase de establecimiento, se tomó a bien un lapso poco mayor a los 30 días. En esta fase, los explantes aumentaron de tamaño y multiplicaron sus yemas.

Las nuevas yemas extraídas de esta fase fueron divididas y seleccionadas para realizar el nuevo establecimiento según tratamientos. La selección se llevó a cabo según tamaño y desarrollo de las yemas para tener una muestras más o menos similares, tanto para el te-

Figura 1. Cuadro descriptivo de materiales. 
rreno de cultivo in vitro Murashige \& Skoog como para el terreno UNICAES 2.

La creación del terreno de cultivo in vitro UNICAES 2 se ha dado como una evolución/ adaptación del terreno UNICAES a las necesidades y requerimientos del cultivo de piña, con especificidad en piña MD-2 (Ananascomosus L. Merril). También para corregir problemas con excesos de elementos presentes en el medio de cultivo.

Después de seguir la formulación del terreno UNICAES 2, este, al igual que el terreno Murashige \& Skoog, se coloca en un autoclave para ser sometido a $121^{\circ} \mathrm{C}$ y a 15 libras de presión por 15 minutos.

La receta UNICAES 2 se detallará más adelante, pero una previa de la misma es:

\begin{tabular}{|c|}
\hline 3 g de 18-6-18-2-8 + EM (hidrosoluble) \\
\hline $3 \mathrm{ml}$ de Bayfolan Forte \\
\hline $0.15 \mathrm{ml}$ de Bio Q \\
\hline $0.44 \mathrm{~g}$ de Cloruro de Calcio dihidratado \\
\hline $1 \mathrm{ml}$ Vitaminas Murashige \& Skoog. \\
\hline 30 g de Azúcar comercial \\
\hline $0.1 \mathrm{~g}$ de Myo Inositol \\
\hline $3 \mathrm{ml}$ de 6 bencil amino purina \\
\hline pH 5.8 \\
\hline $2.2 \mathrm{~g}$ de Phytagel \\
\hline Todo se vació en frascos de $225 \mathrm{ml}, 30 \mathrm{ml} /$ frasco \\
\hline
\end{tabular}

Figura 2. Cuadro descriptivo de fórmula de terreno UNICAES 2.

\section{d. Terreno UNICAES 2}

El terreno de cultivo UNICAES 2 nace de la necesidad de suplir los requerimientos nutricionales del cultivo de piña en procesos in vitro, de una forma económica. La fórmula del mismo es una modificación del terreno de cultivo UNICAES. Este terreno funcionó bien en el desarrollo de musáceas, pero no en piña.

Este terreno está formulado casi en su totalidad por fertilizantes comerciales de fácil acceso, reduciendo drásticamente los costos de fabricación del mismo. (Ver tabla 1).

En la tabla 1 se observan los valores nutritivos de cada compuesto, tomados de los cuadros informativos de los mismos y cuantificados de acuerdo con los niveles de elementos que ofrece el terreno de cultivo Murashige \& Skoog modificado para piña. 
Tabla 1. Formulación del terreno de cultivo UNICAES 2

\begin{tabular}{|c|c|}
\hline Aporte del elemento & Producto comercial \\
\hline Nitrógeno & \\
\hline Potasio & \\
\hline Calcio & \\
\hline Magnésio & \\
\hline Fósforo & \\
\hline Hierro & $3 \mathrm{~g}$ de $18-6-18-2-8+$ EM (hidrosoluble), \\
\hline Boro & $3 \mathrm{ml}$ de Bayfolan Forte, \\
\hline Manganeso & 0.15ml de Bio Q \\
\hline Zinc & $30 \mathrm{~g}$ de azúcar comercial. \\
\hline Yodo & \\
\hline Molibdeno & \\
\hline Cobre & \\
\hline Cobalto & \\
\hline Azúcar & \\
\hline \multicolumn{2}{|c|}{ Productos tradicionales de laboratorio (calidad reactivo) } \\
\hline Calcio & Cloruro de calcio di hidratado $0.44 \mathrm{~g} / \mathrm{l}$ \\
\hline Glicina & \multirow{4}{*}{$1 \mathrm{ml}$ de vitaminas Murashige\&Skoo ${ }^{\circ} \mathrm{g}$} \\
\hline Ácido Nicitínico & \\
\hline Pyridoxina & \\
\hline ThiaminaHCl & \\
\hline MioInositol & $0.1 \mathrm{~g}$ \\
\hline 6 Bencil amino purina & $3 \mathrm{mg} / \mathrm{L}$ \\
\hline $\mathrm{pH}$ & 5.8 \\
\hline
\end{tabular}




\section{e. Desarrollo de las pruebas}

En la segunda fase de multiplicación se llevaron a cabo las pruebas con los tratamientos. En esta fase se dividieron y establecieron 30 pruebas por tratamiento respectivamente. De ellas, solamente 21 pruebas fueron tomadas al azar de cada uno de los tratamientos, las cuales se compararon en respecto a los siguientes criterios: desarrollo de yemas, multiplicación de yemas y longitud de las mismas. Esto con el fin de otorgar cierto grado de uniformidad a las muestras de cada tratamiento.

Después de establecidos los tratamientos, se tomó un margen de tiempo de 35 días para hacer los análisis correspondientes. Se consideró este tiempo como suficiente para hacer notar el efecto de terreno en el desarrollo y crecimiento de las plantas de piña en cada uno de los tratamientos.

\section{Resultados y discusión}

\section{a. Desarrollo de Yemas}

Los análisis estadísticos se llevaron a cabo con la ayuda del programa estadístico StatGraphics, en el cual se vaciaron los valores para comparar el desarrollo de yemas entre los tratamientos.

Puesto que el valor-P es mayor o igual que 0.05 (P valor 0.3050), no existe una diferencia estadísticamente significativa entre la media de tratamientos para la parte de desarrollo de yemas, con un nivel del $95.0 \%$ de confianza. Esto significa que no hay una marcada diferencia entre los resultados con base a desarrollo de yemas obtenidas en el tratamiento 1 y tratamiento 2 .

\section{b. Multiplicación de Yemas}

Como multiplicación de yemas se entiende: el número de yemas obtenidas de una sola yema inicial en un tiempo determinado. Este factor es determinante para la propagación de algún cultivo, muy importante para conocer la rentabilidad de la implementación de uno u otro tratamiento.

Puesto que el valor-P es menor que 0.05 , existe una diferencia estadísticamente significativa entre la media de Número de Yemas entre un nivel de tratamiento y otro, con un nivel del 95.0\% de confianza. Lo anterior respecta a la multiplicación de yemas con una marcada superioridad del medio Murashige \& Skoog por sobre el medio UNICAES 2.

\section{Conclusión}

Las pruebas iniciales se realizaron con el terreno de cultivo UNICAES, el cual presentó niveles tóxicos de fósforo que no pudo soportar la piña, lo cual no había ocurrido con otros cultivos como el de plátano. Dicho medio es adecuado para musáceas de distinto tipo, pero no para piñas. Por otra parte, se dio un crecimiento y respuesta positiva al inicio del 
proceso de establecimiento, pero luego los explantes se debilitaron, tornaron su color verde a un verde claro muy pálido; sus células se encontraban claramente afectadas en su nivel de rigidez y sostén. Esto volvía inviables los resultados iniciales obtenidos con este medio. Este terreno de cultivo tuvo que ser eliminado por los problemas que presentó.

Después de meses de pruebas y modificaciones de los componentes del terreno de cultivo UNICAES, para la adaptabilidad en piña, se consiguió el desarrollo de un nuevo terreno de cultivo in vitro llamado UNICAES 2. Este reducía drásticamente la cantidad de fósforo presente en el medio, pero con la ventaja de no llegar a un déficit del mismo.

Las pruebas se llevaron a cabo contrarrestando los resultados obtenidos con el terreno de cultivo in vitro Murashige \& Skoog, y los obtenidos con el terreno de cultivo in vitro UNICAES 2.

Se corrió un análisis estadístico en el programa StatGraphics para conocer más certeramente los resultados comparativos de ambos tratamientos en número de yemas y desarrollo de las mismas. Los resultados arrojados por el programa indicaron un análisis fidedigno y permitió conocer las ventajas de cada terreno de cultivo en comparación con el otro tratamiento.
La funcionalidad del terreno de cultivo UNICAES 2 fue positiva a la hora de analizar el desarrollo de yemas de piña, sin encontrarse diferencias significativas con el uso del medio Murashige \& Skoog para este propósito. La diferencia entre ambos es estadísticamente mínima. Por otro lado, si se encontró una diferencia significativa entre los medios de cultivo in vitro para la multiplicación de yemas, encontrando superior los resultados del terreno Murashige \& Skoog por sobre los resultados del terreno UNICAES 2. Este factor es de vital importancia para la determinación de la rentabilidad de la implementación de uno u otro terreno de cultivo.

Visto que el terreno de cultivo Murashige \& Skoog es superior a la hora de esperar una buena multiplicación de yemas, es recomendable la utilización el terreno UNICAES 2 para las fases últimas del cultivo de piña in vitro, donde sólo se busca un buen desarrollo de los explantes para pasar al proceso de aclimatación. Esto puede reflejarse como una disminución en el uso de reactivos calidad de laboratorio en el proceso completo de piña in vitro, lo cual se convierte en beneficios económicos para las empresas que deseen aplicarlos.

El terreno de cultivo in vitro UNICAES 2 demuestra la factibilidad de hacer pruebas para la creación de terrenos de cultivo a base de fertilizantes comerciales, que aporten los nutrientes necesarios a los cultivos y colaboren 
con la reducción de costos de fabricación y el consecuente aumento de beneficios económicos. Se recomienda la realización de más pruebas a fin de perfeccionar el terreno de cultivo UNICAES 2 y así hacerlo factible para más cultivos.
Debido a la importancia creciente que obtiene la biotecnología para la creación de alimentos de alta calidad, se recomienda la realización de posteriores investigaciones para profundizar los resultados.

\section{Referencias}

Alfaro, G. (2011). Piña: Ficha de mercadeo Nº4. Ministerio de Agricultura y Ganadería. Recuperado de: file:///C:/Users/J.\%20F.\%20Cuellar/Downloads/pia.pdf.

Cerrato, I. (2013). Parámetros de comercialización de la piña MD2 en los principales mercados hondureños. Secretaria de Agricultura y Ganadería. Programa Nacional de Desarrollo Agroalimentario. PDF en línea. Recuperado de: file://C:/Users/J.\%20F.\%20Cuellar/Downloads/ PARAMETROS-DE-COMERCIALIZACION-DE-LA-PINA-MD2.pdf

Costa Rica, Ministerio de Agricultura y Ganadería (1991). Piña, tomado de libro: Aspectos Técnicos sobre Cuarenta y Cinco Cultivos Agrícolas de Costa Rica. Dirección General de Investigación y Extensión Agrícola. Costa Rica. Recuperado de: http://www.mag.go.cr/bibioteca_virtual_ciencia/tec-pina.pdf

Pac, S. Pedro J. (2005). Experiencias en el cultivo de piña (Annanas Comosus (L) Merr.) Con el hibrido MD2 en finca La Plata, Coatepeque, Quetzaltenango. Universidad de San Carlos de Guatemala, Facultad de Agronomía, Instituto de Investigaciones Agronómicas. Guatemala. Recuperado de: http://biblioteca.usac.edu.gt/tesis/01/01_2161.pdf

Pierik, R.L.M. (1990) Cultivo in vitro de las plantas superiores. Versión española de MateoSagasta, A. Madrid, España. Ediciones Muni-Prensa.

Roca, W. Mroginski, L. et al. (1991) Cultivo de Tejidos en la Agricultura: Fundamentos y Aplicaciones. CIAT. Cali, Colombia. 
Silvia, S. et al. (2008) Propagación Clonal in vitro de Piña (Ananas comosus L. Merr) Variedades Champaka y Hawaiana Universidad Estatal Técnica de Quevedo. Los Ríos, Ecuador. Recuperado de: http://www.uteq.edu.ec/revista_cyt/archivos/2008/v1/articulo_9.pdf

Suárez, F. (2011). Microporpagación in vitro de piña (Ananas comosus L. Merril) a partir de yemas laterales y apicales. (Tesis de ingeniería, Escuela Polotécnica del Ejército). Recuperado de: http://repositorio.espe.edu.ec/bitstream/21000/4952/1/T-ESPE-IASA\%20I-004581.pdf

Uriza Á., D. E. (2011). Programa estratégico para el desarrollo rural sustentable de la región sursureste de México: Trópico Húmedo. Paquete tecnológico piña MD2. Establecimiento y mantenimiento. Centro Regional de Investigación Golfo Centro. Veracruz: Campo experimental Cotaxtla/Papaloapan. Recuperado de: www.inifap.gob.mx/Documents/inicio/paquetes/pina.pdf 